\title{
Lack of Plasma-like Screening Mechanism in Sedimentation of a Non-Brownian Suspension
}

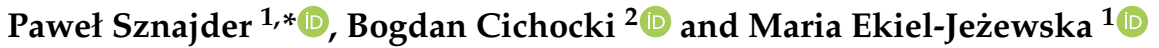 \\ 1 Institute of Fundamental Technological Research, Polish Academy of Sciences, 02-106 Warsaw, Poland; \\ mekiel@ippt.pan.pl \\ 2 Faculty of Physics, University of Warsaw, 02-093 Warsaw, Poland; bogdan.cichocki@fuw.edu.pl \\ * Correspondence: psznaj@ippt.pan.pl
}

Citation: Sznajder, P.; Cichocki, B.; Ekiel-Jeżewska, M. Lack of Plasma-like Screening Mechanism in Sedimentation of a Non-Brownian Suspension. Symmetry 2022, 14, 63. https://doi.org/10.3390/ sym 14010063

Academic Editor: Andrzej Herczyński and Roberto Zenit

Received: 19 November 2021 Accepted: 17 December 2021 Published: 3 January 2022

Publisher's Note: MDPI stays neutral with regard to jurisdictional claims in published maps and institutional affiliations.

Copyright: (C) 2022 by the authors. Licensee MDPI, Basel, Switzerland. This article is an open access article distributed under the terms and conditions of the Creative Commons Attribution (CC BY) license (https:// creativecommons.org/licenses/by/ $4.0 /)$.

\begin{abstract}
We investigate qualitatively a uniform non-Brownian sedimenting suspension in a stationary state. As a base of our analysis we take the BBGKY hierarchy derived from the Liouville equation. We then show that assumption of the plasma-like screening relations can cancel some long-range terms in the hierarchy but it does not provide integrable solutions for correlation functions. This suggests breaking the translational symmetry of the system. Therefore a non-uniform structure can develop to suppress velocity fluctuations and make the range of correlations finite.
\end{abstract}

Keywords: non-Brownian sedimentation; stability; BBGKY hierarchy; hydrodynamic screening; correlation functions; low-Reynolds-number hydrodynamics

\section{Introduction}

Sedimentation is a process of falling of particles in a fluid due to gravity (the particles are more dense then the fluid). Considerations in this paper are restricted to the limit of vanishing Reynolds number and infinite Peclet number. Reynolds number is given by

$$
\operatorname{Re}=a \eta^{-1} \rho_{l} U_{S}
$$

where $a$ is radius of a particle, $\eta$ is dynamic viscosity coefficient of the fluid, $\rho_{l}$ is density of the fluid and $U_{S}$ is the Stokes velocity of a single particle falling in unbounded fluid motionless at infinity. When $\operatorname{Re} \rightarrow 0$, the fluid flow instantly adjusts to the boundary conditions. Peclet number is given by

$$
\mathrm{Pe}=a D^{-1} U_{S},
$$

where $D$ is diffusion coefficient of a single particle in a fluid. In case of $\mathrm{Pe} \rightarrow \infty$, Brownian motion is negligible compared to the motion caused by gravity and hydrodynamic interactions. In such a system there are difficulties with divergent expressions due to hydrodynamic field disturbance slowly decaying over distance (inversely proportional to distance-same as electric potential produced by an isolated charge in a vacuum). Challenges of theoretical approach to system with vanishing Reynolds number (and divergent Peclet number) are known for more than hundred years. In 1911, Smoluchowski [1,2] investigated a particle surrounded by other particles suspended in a Newtonian fluid. His observation was that, considering larger and larger systems leads to a divergent expression for the particle falling velocity, caused by the long-range velocity disturbance produced by other particles. Now this is known in literature as the Smoluchowski paradox [3]. Solution of this paradox was given by Batchelor [4] sixty years after the work of Smoluchowski and then reanalyzed by Beenakker and Mazur [5].

Batchelor's main idea was to calculate the average velocity of suspended particles $\langle U\rangle$ relative to the flow $\langle v\rangle$ of the whole suspension which resulted in $\langle U\rangle-\langle v\rangle$ where cancellation of divergent terms secures that the average relative velocity is finite. Nevertheless 
there is another problem within physics of such a system: calculation of the sedimentation velocity variance. This one was noticed by Caflisch and Luke [6] in 1985. They showed that fluctuations in general should grow with linear dimension $L$ of a system which is again a result of a slowly decaying flow disturbance around a particle. One can spot that if this flow disturbance is inversely proportional to distance then integrating square of it leads to linear divergence with $L$. To solve that conundrum, Koch and Shaqfeh [7] proposed in 1991 that pair probability has to obey the screening relation like in plasma (as it is with the Debye-Hückel screening), namely that it has to reflect a net deficit of one particle in the vicinity of each particle. This condition corresponds to vanishing structure factor for vanishing $k$ wave vector, $S(k \rightarrow 0)=0$. The idea of screening is that hydrodynamic interaction between particles results in lower probability of finding a close pair of particles. When screening occurs this effect is finely tuned in such a way that effective hydrodynamic interaction between particles within a pair has a much faster decay with the inter-particle distance-a power law is replaced by an exponential decay. Moreover, the change is such that integral of the correlation function reflects the average lack of exactly one neighboring particle around a test particle. This leads to cancellation of the leading long-range terms and results in convergent expressions for velocity fluctuations. An example of such a cancellation owing to the screening effect will be provided in Section 3.1.

Nevertheless, the idea of the screening condition being satisfied was a subject of a substantial critique [8-11] which was mainly focused on showing no evidence of screening in simulations of a sedimenting suspension. In order to cope with fluctuations many ideas were given. Some were pointing to the crucial role of walls [12], some were focusing on stratification [13] or its critique [14,15], some were considering additional shear flow [16], and others even tried to look for a solution in non-zero Re number [17]. Good scope of troubles with finding solution to fluctuation problem is given in review articles [18-20].

In this paper, the screening idea is analyzed and it is shown that the BBGKY (Bogoliubov, Born, Green, Kirkwood, Yvon) hierarchy for the correlation functions between sedimenting particles [21] in a uniform stationary state has no screening solutions at all. This is a significant problem since BGGKY hierarchy is derived straightforwardly from the Liouville equation which cannot be violated.

In the Section 2, the theoretical framework is presented. In Section 3 it is shown that even if some of long range terms in hierarchy are regularized by screening conditions, there are still other ones which cannot be screened at all. This leads to contradiction since assuming screening still leads to equations which give non-integrable solutions for the correlation functions. This suggests that the system becomes non-uniform and symmetry with respect to translations is broken. As pointed out in Section 4, this finding gives a novel, unique theoretical explanation of a non-uniform structure of non-Brownian sedimenting suspensions, previously reported in experiments and numerical simulations [13,19,22-32].

\section{Methods}

\subsection{System and Its Theoretical Description}

System consists of $N$ identical rigid spherical particles (mono-disperse suspension) immersed in a Newtonian fluid of viscosity $\eta$. Particle radius $a$ is small enough and viscosity of fluid is sufficiently large to ensure Reynolds number $(\operatorname{Re})$ is much smaller than unity. Then one can set $\operatorname{Re}=0$ which reduces Navier-Stokes equations for the fluid flow to the Stokes equations [33]. Simultaneously $a$ is large enough to keep Peclet number (Pe) much larger then 1 so Brownian motion can be neglected. Particles are uniform, spherically symmetric and their density $\rho_{p}$ is larger than the density $\rho_{f}$ of the surrounding fluid, which results in settling of those particles under gravity. Macroscopically system is assumed to be uniform and stationary. 
The fluid flow is governed by the Stokes equations. The Green tensor for an unbounded system, with condition that fluid velocity is zero at infinity, is represented by the Oseen tensor $\mathcal{T}$ of the form [34]:

$$
\mathcal{T}(\boldsymbol{r})=\frac{1}{8 \pi \eta r}(\mathbb{I}+\hat{\boldsymbol{r}} \hat{\boldsymbol{r}}) .
$$

Velocities of the fluid and particles are determined by external forces (By external forces one should understand those which are not a result of an interaction with the fluid) and boundary conditions at infinity and at the particles. It is important to solve the so-called mobility problem which is stated as below: determine velocities of the particles given their positions and total external forces acting upon them, in the absence of any external torques on the particles. The solution is represented by $3 \times 3$ Cartesian mobility matrices $\mu_{i j}$, with the particle labels $i, j=1, \ldots, N$ [35],

$$
\boldsymbol{U}_{i}(\boldsymbol{X})=\sum_{j=1}^{N} \boldsymbol{\mu}_{i j}(\boldsymbol{X}) \boldsymbol{F}_{j}
$$

where

$$
\boldsymbol{X}=\left\{\boldsymbol{R}_{1}, \ldots, \boldsymbol{R}_{N}\right\}
$$

is the set of positions $\boldsymbol{R}_{i}$ of all the particle centers, $\boldsymbol{U}_{i}$ is the velocity of $i$-th particle, $\boldsymbol{F}_{j}$ is the external force, e.g., gravity, acting on particle $j$. In this paper all the forces are identical, $\boldsymbol{F}_{j}=\boldsymbol{F}$. In general, mobility matrices $\boldsymbol{\mu}_{i j}$ depend on positions of all the particles $X$. Although $\boldsymbol{\mu}_{i j}$ have many-body character, they can be expressed as an infinite sum of sequences of two-particle interactions. Such a scattering expansion [36-39], analogous to the reflection method [33], can be written as,

$$
\boldsymbol{\mu}_{i j}=\left(\boldsymbol{\mu}_{\mathbf{0}}+\boldsymbol{\mu}_{\mathbf{0}} \mathcal{Z}_{\mathbf{0}} \mathcal{G}\left[\sum_{k=0}^{\infty}\left(-\hat{\mathcal{Z}}_{\mathbf{0}} \mathcal{G}\right)^{k}\right] \mathcal{Z}_{\mathbf{0}} \boldsymbol{\mu}_{\mathbf{0}}\right)_{i j}
$$

At the RHS of the above equation, we use the operators, defined, e.g., in $[38,40,41]$ and briefly described below. The single particle operators: mobility $\boldsymbol{\mu}_{0}$, friction $\mathcal{Z}_{\mathbf{0}}$ and convective friction $\hat{\mathcal{Z}}$ are diagonal in the particle labels $i$, and identical for each value of $i$ because the particles are identical. Their meaning is the following,

$$
\begin{aligned}
\mu_{0}: & \text { force on a particle } \mapsto \text { velocity of that particle } \\
\mathcal{Z}_{0}: & \text { incoming fluid velocity field at a particle } \\
& \mapsto \text { force density induced on that particle } \\
\hat{\mathcal{Z}}_{0}: & \text { incoming fluid velocity field at a freely moving particle } \\
& \mapsto \text { force density induced on that particle }
\end{aligned}
$$

The difference between the operators $\mathcal{Z}_{0}$ and

$$
\hat{\mathcal{Z}}_{0}=\mathcal{Z}_{0}-\mathcal{Z}_{0} \mu_{0} \mathcal{Z}_{0}
$$

is that in case of $\mathcal{Z}_{0}$, the particle translational and rotational velocities are given, while in case of $\hat{\mathcal{Z}}_{0}$, the particle moves freely, what by definition means that the total induced force and torque on that particle vanish $[38,40,41]$.

The off-diagonal operator $\mathcal{G}$ contains as its elements propagators based on the Oseen tensor given by Equation (3). The elements $\mathcal{G}_{i j}$ are propagators between different particles $i \neq j$, and they represent single hydrodynamic interactions. The element $\mathcal{G}_{i j}$ depends only on the relative position $\boldsymbol{R}_{i j}=\boldsymbol{R}_{i}-\boldsymbol{R}_{j}$, and it acts in the following way,

$$
\mathcal{G}_{i j}\left(\boldsymbol{R}_{i j}\right) \text { : force density on } j \text {-th particle at } \boldsymbol{R}_{j} \mapsto \text { generated fluid velocity at } \boldsymbol{R}_{i}
$$


In general, the operators $\mu_{0}, \mathcal{Z}_{0}, \hat{\mathcal{Z}}_{0}$ and $\mathcal{G}$ are complicated but one can simplify description by expressing those elements in terms of vector solid harmonics [34] as base functions given by irreducible representations of the $\mathrm{SO}(3)$ group. It results in multipole expansion of hydrodynamic interactions [33,42-45] which is one of among other successful applications of multipole formalism like in electromagnetism [46] or gravity [47]. The base multipole functions are characterized by three indices $l, m, \sigma$, with $l=1,2, \ldots, m=-l, \ldots, l$ and $\sigma=0,1,2$. Using them, one can represent each operator as a large matrix with the particle labels and the multipole indices, $i l m \sigma$. In this way, superposition of the operators in Equation (6) should be understood as multiplication of the corresponding large matrices. In particular, $\mathcal{G}_{i l m \sigma, j l^{\prime} m^{\prime} \sigma^{\prime}}$ describes the coupling between $l^{\prime} m^{\prime} \sigma^{\prime}$ force multipole on $j$-th particle and $l m \sigma$ velocity multipole on $i$-th particle. Using the multipole representation is very convenient for asymptotic analysis since the dependence of $\mathcal{G}$ elements on distance is very simple [44],

$$
\mathcal{G}_{i l m \sigma, j l^{\prime} m^{\prime} \sigma^{\prime}} \propto R_{i j}^{-l-l^{\prime}-\sigma-\sigma^{\prime}+1} .
$$

To proceed with further analysis it is needed to point out the crucial difference between the multipole elements of $\mathcal{Z}_{0}$ and $\hat{\mathcal{Z}}_{0}$. Both operators are diagonal in pairs of indices $l, l^{\prime}$, and $m, m^{\prime}$. For $l \geq 2$ their multipole elements are identical. However, they are different for $l=1$. In this case, the operator $\hat{\mathcal{Z}}_{0}$ has the only non-zero element for $\left(\sigma, \sigma^{\prime}\right)=(2,2)$, determined by the relation (10).

Therefore, when $\mathcal{G}_{i j}$ is between operators $\hat{\mathcal{Z}}_{0}$ in a scattering sequence given by Equation (6), the slowest possible decay of the interaction is for $\left(l, l^{\prime}\right)=(2,2)$ and $\left(\sigma, \sigma^{\prime}\right)=(0,0)$ which, according to Equation (12), scales as $1 / R_{i j}^{3}$. This specific term corresponds to coupling between symmetric vector dipole moments $(l=2, \sigma=0)$ so it can be called a dipole-dipole interaction.

Using the multipole representation, it is now easier to understand the scattering expansion (6). Taking into account that the large matrices representing the single particle operators $\mu_{0}, \mathcal{Z}_{0}$ and $\hat{\mathcal{Z}}_{0}$ are diagonal in the particle labels, and the diagonal elements are identical for each particle, from now on we will redefine the symbols $\mu_{0}, \mathcal{Z}_{0}$ and $\hat{\mathcal{Z}}_{0}$ to denote the corresponding diagonal elements, which are matrices in the space of the multipole indices. In the new notation, the first term in the bracket at the RHS of (6) is $\mu_{0} \delta_{i j}$. The second term is the single-scattering sequence $\mu_{0} \mathcal{Z}_{0} \mathcal{G}_{i j} \mathcal{Z}_{0} \mu_{0}$, with multiplication of the consecutive multipole matrices. The third term is the sum of the two-scattering sequences, $-\mu_{0} \mathcal{Z}_{0} \mathcal{G}_{i m} \hat{\mathcal{Z}}_{0} \mathcal{G}_{m j} \mathcal{Z}_{0} \mu_{0}$, with respect to $m \neq i$ and $m \neq j$, and so on.

\subsection{BBGKY Hierarchy of Equations for Correlation Functions}

To derive the BBGKY hierarchy of equations for the correlation functions of sedimenting particles one needs to consider the Liouville equation for the system. For a stationary state it reads [21]:

$$
\sum_{i=1}^{N} \frac{\partial}{\partial \boldsymbol{R}_{i}} \cdot\left[\boldsymbol{U}_{i}(\boldsymbol{X}) \rho(\boldsymbol{X})\right]=0 .
$$

where $\rho(\boldsymbol{X})$ is the probability distribution function for particle positions. To proceed further one needs to introduce $m$-particle microscopic density operator

$$
\hat{n}_{m}(\boldsymbol{m}, \boldsymbol{X})=\sum_{j_{1}, j_{2}, \ldots, j_{m}} \prod_{i=1}^{m} \delta\left(\boldsymbol{r}_{i}-\boldsymbol{R}_{j_{i}}\right)
$$

where $m$ is set of positions

$$
\boldsymbol{m}=\left\{\boldsymbol{r}_{1}, \ldots, \boldsymbol{r}_{m}\right\},
$$

also abbreviated to $\{1, \ldots, m\}$. The sum $\Sigma^{\prime}$ is taken over all $m$-particle subsets and their permutations which results in sums with respect to $j_{i}$ where $i=1, \ldots, m$ and there is no 
duplicating indices $\forall_{i, k} j_{i} \neq j_{k}$ (see equation (3.1.20) in [48]). When averaged over positions $\boldsymbol{X}$ one obtains $m$-particle density functions

$$
n_{m}(\boldsymbol{m})=\int \mathrm{d} \boldsymbol{X} \rho(\boldsymbol{X}) \hat{n}_{m}(m, \boldsymbol{X})=\left\langle\hat{n}_{m}\right\rangle,
$$

with

$$
\langle\ldots\rangle=\int \mathrm{d} X \rho(X) \ldots
$$

In case of identical particles one can also recover the well-known formula,

$$
n_{m}(\boldsymbol{m})=\frac{N !}{(N-m) !} \int \mathrm{d} \boldsymbol{X} \rho(\boldsymbol{X}) \delta\left(\boldsymbol{r}_{1}-\boldsymbol{R}_{1}\right) \cdots \delta\left(\boldsymbol{r}_{m}-\boldsymbol{R}_{m}\right) .
$$

Averaging for $m=1$ one gets concentration $n_{1}(1)=n=N / V$ where $N$ is the number of particles and $V$ is the volume of the system. Following [48], we introduce $k$-particle correlation functions $h_{k}(\boldsymbol{k})$, defining them by the relations,

$$
\begin{aligned}
& n_{2}(1,2)= n^{2}\left(1+h_{2}(1,2)\right) \\
& n_{3}(1,2,3)=n^{3}\left(1+h_{2}(1,2)+h_{2}(2,3)+h_{2}(1,3)+h_{3}(1,2,3)\right) \\
& n_{4}(1,2,3,4)=n^{4}\left(1+h_{2}(1,2)+h_{2}(1,3)+h_{2}(1,4)+h_{2}(2,3)+h_{2}(2,4)+h_{2}(3,4)\right. \\
& \quad+h_{2}(1,2) h_{2}(3,4)+h_{2}(1,3) h_{2}(2,4)+h_{2}(1,4) h_{2}(2,3) \\
&\left.+h_{3}(1,2,3)+h_{3}(1,2,4)+h_{3}(2,3,4)+h_{4}(1,2,3,4)\right)
\end{aligned}
$$

Now multiplying the Liouville Equation (13) by density operator $\hat{n}_{m}$ and averaging over all positions $X$ results in generation of the following BBGKY hierarchy of equations for $\mathrm{m}$-particle density functions (It can be transformed to connected hierarchy for correlation functions [21], but for the purpose of this work it is enough to consider this hierarchy as it is.):

$$
\sum_{i=1}^{m} \frac{\partial}{\partial r_{i}} \cdot\left\langle\boldsymbol{U}_{i} \hat{n}_{m}\right\rangle(\boldsymbol{m})=0, \quad \text { with } m=2,3, \ldots
$$

Explicitly,

$$
\begin{array}{r}
\frac{\partial}{\partial r_{1}} \cdot\left\langle\boldsymbol{U}_{1} \hat{n}_{2}\right\rangle(\mathbf{2})+\frac{\partial}{\partial r_{2}} \cdot\left\langle\boldsymbol{U}_{2} \hat{n}_{2}\right\rangle(\mathbf{2})=0 \\
\frac{\partial}{\partial r_{1}} \cdot\left\langle\boldsymbol{U}_{1} \hat{n}_{3}\right\rangle(\mathbf{3})+\frac{\partial}{\partial r_{2}} \cdot\left\langle\boldsymbol{U}_{2} \hat{n}_{3}\right\rangle(\mathbf{3})+\frac{\partial}{\partial r_{3}} \cdot\left\langle\boldsymbol{U}_{3} \hat{n}_{3}\right\rangle(\mathbf{3})=0 \\
\frac{\partial}{\partial r_{1}} \cdot\left\langle\boldsymbol{U}_{1} \hat{n}_{4}\right\rangle(\mathbf{4})+\frac{\partial}{\partial r_{2}} \cdot\left\langle\boldsymbol{U}_{2} \hat{n}_{4}\right\rangle(\mathbf{4})+\frac{\partial}{\partial r_{3}} \cdot\left\langle\boldsymbol{U}_{3} \hat{n}_{4}\right\rangle(\mathbf{4})+\frac{\partial}{\partial r_{4}} \cdot\left\langle\boldsymbol{U}_{4} \hat{n}_{4}\right\rangle(\mathbf{4})=0
\end{array}
$$

For two identical particles, the LHS of Equation (23) is always zero due to the symmetry with respect to interchanging the particle labels, and the translational symmetry of the system. In case of two different particles, Equation (23) was solved by Batchelor [49,50], with the use of the expansion in powers of the concentration $n$. In case of three particles Cichocki and Sadlej [51] proposed a scheme to deal with the long range terms, but limited to mono-disperse case. The problem of long-range terms in Equations (24) and (25), and also higher order equations, will be discussed in the next section. 


\section{Results}

\subsection{Long-Range Terms and Screening}

Hierarchy of the Equations (22) describes the conditions for particle current divergences which represent the law of mass conservation. By considering an increasing number of particles and performing the cluster analysis [21,39] one can obtain equations for the correlation functions. In these equations, there appear long-range terms which scale with the inter-particle distances $r$ as $1 / r, 1 / r^{2}$ and $1 / r^{3}$ which makes them non-integrable in the thermodynamic limit, defined as $N, V \rightarrow \infty$ and $n=N / V=$ const. Without imposing additional conditions, this would lead to non-integrable correlation functions. However, integrability of the correlation functions is necessary for stability of the system. As it is mentioned in the introduction, Koch and Shaqfeh proposed a screening mechanism for the system under consideration, similar as in a one component plasma, as condition (2.14) in [7]. This condition, formulated in terms of the pair probability $g$, can be written in terms of the two-particle correlation function $h_{2}=g / n-1$ as:

$$
n \int \mathrm{d} \mathbf{r} h_{2}(\mathbf{r})=-1
$$

For the higher order correlation functions this should be generalized to [52]:

$$
n \int \mathrm{d} \mathbf{r}_{k+1} h_{k+1}\left(\mathbf{r}_{1}, \mathbf{r}_{2}, \ldots, \mathbf{r}_{k+1}\right)=-k h_{k}\left(\mathbf{r}_{1}, \ldots, \mathbf{r}_{k}\right), \quad k=2,3, \ldots
$$

Those conditions will lead to finite velocity fluctuations in the thermodynamic limit. One can try to apply the conditions (26) and (27) to eliminate (regularize) long-range terms also in the BBGKY hierarchy. However, as it will be shown below, the screening conditions eliminate many but not all long-range terms in the hierarchy. Nevertheless, the screening conditions (26) and (27) can be satisfied only if all the correlation functions have a finite range. Therefore, we will face a contradiction.

In the following, we will first provide an example how the screening condition (26) leads to cancellation of some long-range terms. Then we will show another example of long-range terms which remain unaffected by screening.

Equations (24) and (25), . . of the BBGKY hierarchy for three, four and more particles contain long-range terms. We will focus on Equation (24) and identify such terms. To this goal, one should do substitutions using the expressions (4) for the particle velocities $\boldsymbol{U}_{i}$ in terms of the mobilities $\boldsymbol{\mu}_{i j}$, given as the series (6) of scattering sequences. Each sequence linking particles $i$ and $j$ consists of a certain number of scatterings, generated by the force $\boldsymbol{F}$ acting on particle $j$. The scattering sequences are next averaged, what leads to multiplication by certain correlation functions. It is useful to remember that the density operator $\hat{n}_{m}$ sets positions of particles $1,2, \ldots, m$ regardless whether they are included in a particular scattering sequence for $\boldsymbol{U}_{i}$ or not, with $\boldsymbol{U}_{i}$ given by Equations (4) and (6). It means that if there are particle positions in a certain BBGKY hierarchy term $\left\langle\boldsymbol{U}_{i} \hat{n}_{m}\right\rangle(\boldsymbol{m})$ which are being integrated then they must appear in a scattering sequence of $\boldsymbol{U}_{i}$.

We will now provide the first example. In the expression (24) there exists a term with the single interaction $\mathcal{G}_{12}$ between particles 12 , the single interaction $\mathcal{G}_{23}$ between particles 23 and the pair correlation $h_{2}\left(\boldsymbol{r}_{12}\right)$ between the particles 12 . This term can be written as follows,

$$
n^{3} \frac{\partial}{\partial \boldsymbol{r}_{1}} \cdot\left[\boldsymbol{\mu}_{\mathbf{0}} \mathcal{Z}_{0} \mathcal{G}_{12} \hat{\mathcal{Z}}_{0} \mathcal{G}_{23} \mathcal{Z}_{0} \mu_{0} F h_{2}\left(\boldsymbol{r}_{12}\right)\right]
$$

The slowest decaying part of this term is proportional to $r_{23}^{-2}$ since it is between operators $\mathcal{Z}_{0}$ and $\hat{\mathcal{Z}}_{0}$, and we know how $\mathcal{G}_{23}$ can vary with distance given (12). If $n \int \mathrm{d} \mathbf{r} h_{2}(\mathbf{r})=$ -1 holds then the expression (28) can be screened by another term from Equation (24), involving in addition a fourth particle and containing integration with respect to its position $\boldsymbol{r}_{4}$. This term contains the single interaction $\mathcal{G}_{12}$ between particles 12 , the single interaction 
$\mathcal{G}_{24}$ between particles 24 , the pair correlation functions $h_{2}\left(r_{12}\right)$ and $h_{2}\left(r_{34}\right)$ between pairs of particles 12 and 34,

$$
n^{4} \frac{\partial}{\partial r_{1}} \cdot\left[\int \mathrm{d} r_{4} \mu_{0} \mathcal{Z}_{0} \mathcal{G}_{12} \hat{\mathcal{Z}}_{0} \mathcal{G}_{24} \mathcal{Z}_{0} \mu_{0} F h_{2}\left(r_{12}\right) h_{2}\left(r_{34}\right)\right] .
$$

In this term $\mathcal{G}_{24}$ can be expanded in the following way,

$$
\mathcal{G}_{i k}=\mathcal{G}_{i j}+\boldsymbol{r}_{j k} \cdot \frac{\partial}{\partial r_{i j}} \mathcal{G}_{i j}+\ldots,
$$

with $i, j, k=2,3,4$. Such an expansion, when plugged into Equation (29), is justified since, according to our assumption, the correlation function $h_{2}\left(r_{34}\right)$ has short range. When taking into account the first term of (30), the expression (29) reads

$$
n^{3} \frac{\partial}{\partial r_{1}} \cdot\left[\mu_{0} \mathcal{Z}_{0} \mathcal{G}_{12} \hat{\mathcal{Z}}_{0} \mathcal{G}_{23} \mathcal{Z}_{0} \mu_{0} F h_{2}\left(\boldsymbol{r}_{12}\right) n \int \mathrm{d} r_{4} h_{2}\left(r_{34}\right)\right] .
$$

with the screening condition (26), the expressions (28) and (31) cancel each other. The second term of (30) gives no contribution to expression (29) because, due to symmetry of correlation function, $\int r h_{2}(r) \mathrm{d} r=0$. The correlation function has to obey general symmetry of the system, first one with respect to the rotation around vertical axis and second one with respect to parity in the direction of that axis. Higher order terms in (30) lead in Equation (29) to the hydrodynamic interaction between particles 2 and 3 which decays at least as fast as $r_{23}^{-4}$ so it is then of a short range. Ability to construct a screening pair of terms is based on the fact that those screened interactions are the first from the right in the scattering sequence.

Such a construction is not possible otherwise which gives rise to examples contradicting the possibility of screening of all terms. An example of such term can be found in the hierarchy Equation (25) for 4 particles. This term is written below,

$$
n^{4} \frac{\partial}{\partial r_{1}} \cdot\left[\mu_{0} \mathcal{Z}_{0} \mathcal{G}_{12} \hat{\mathcal{Z}}_{0} \mathcal{G}_{23} \hat{\mathcal{Z}}_{0} \mathcal{G}_{34} \mathcal{Z}_{0} \mu_{0} F h\left(r_{12}\right) h\left(r_{34}\right)\right]
$$

Above, the expression $\hat{\mathcal{Z}}_{0} \mathcal{G}_{23} \hat{\mathcal{Z}}_{0}$ decays as $r_{23}^{-3}$ and therefore corresponds to infinite range of interaction as it was explained below Equation (12). In analogous way to formation of the pair (28) and (31) the term (32) can be paired with the following one,

$$
n^{5} \frac{\partial}{\partial r_{1}} \cdot\left[\int \mathrm{d} r_{5} \mu_{0} \mathcal{Z}_{0} \mathcal{G}_{12} \hat{\mathcal{Z}}_{0} \mathcal{G}_{25} \hat{\mathcal{Z}}_{0} \mathcal{G}_{54} \mathcal{Z}_{0} \mu_{0} F h\left(r_{12}\right) h_{3}\left(\boldsymbol{r}_{34}, \boldsymbol{r}_{35}\right)\right] .
$$

In this case, one has to expand two hydrodynamic interaction propagators, $\mathcal{G}_{25}$ and $\mathcal{G}_{54}$ using (30), with $i, j, k$ equal to 2,3,5 and 5,3,4 respectively. However, this operation together with the screening condition (27) for $k=2$ does not lead to cancellation of infinitely long range interactions like it was with a pair of (28) and (31).

In general, such a dipole-dipole part of the hydrodynamic interaction $\mathcal{G}_{i j}$ inside a scattering sequence, which gives rise to long range behavior of correlations, cannot be screened by the plasma-like screening conditions (27). This is due to the fact that construction of a screening pair requires also expanding preceding interaction which is on the right side of $\mathcal{G}_{i j}$. This situation results in an inability to cancel the slowest $r_{i j}^{-3}$ term because one gets integrals which contains both correlations and interactions and they cannot be reduced by screening conditions. It means that screening can only affect first from right interaction in the scattering sequence. Therefore, only some long range terms are canceled by (27). Other ones still remain, as illustrated in the above example. This leads to a contradiction with the assumption that correlation functions are integrable.

This means that one will not obtain for correlations an integrable solution of the BBGKY hierarchy. This contradicts screening relations leading to instability of a uniform 
system due to divergent expressions for velocity fluctuations in the thermodynamic limit. It suggest that system has to break the translational symmetry and that it has to develop some non-uniform structure like stratification or swirls [22] which can change the velocity fluctuations magnitude [13]. However, for numerical simulations of small systems in periodic boundary conditions $[9,53]$ fluctuations are small enough to keep the stationary state uniform. In those systems mechanism proposed by Cichocki and Sadlej [51] to regularize long range interactions may be a hint to find a solution for correlation functions.

\title{
4. Conclusions
}

In this article, it has been shown that in the BBGKY hierarchy [21] for the correlation function in a sedimenting uniform non-Brownian suspension there is no solution that obeys plasma-like screening relations. This was done by assuming validity of screening conditions and showing that this hypothesis cannot eliminate all long-range terms in the BBGKY hierarchy, and therefore it leads to a contradiction with our assumption. Our results suggest that there is no uniform steady state in the thermodynamic limit. The problem cannot be solved by a system invariant to translations. Our finding is that the uniformity is broken at least on a certain length scale $[19,22,23]$. This seems to be in an agreement with experimental and numerical investigations. They show that non-uniform structures like swirls [13,24-29] or stratification [30-32] are likely to develop in sedimenting suspensions.

\begin{abstract}
Author Contributions: Conceptualization, B.C.; investigation, P.S., B.C. and M.E.-J.; formal analysis, P.S.; writing—original draft preparation, P.S.; writing—review and editing, P.S., M.E.-J. and B.C.; funding acquisition, M.E.-J. All authors have read and agreed to the published version of the manuscript.
\end{abstract}

Funding: P.S. and M.E.-J. were supported in part by the National Science Centre under grant UMO2018/31/B/ST8/03640.

Conflicts of Interest: The authors declare no conflicts of interest.

\section{References}

1. Smoluchowski, M. Über die Wechselwirkung von Kugeln, die sich in einer zähen Flüssigkeit bewegen(Mutual interaction of spheres in a viscous medium) 28-29. Bull. Int. Acad. Sei. Cracovie 1911, 2, 182-194.

2. Smoluchowski, M. On the practical applicability of Stokes law of resistance and its modifications required in certain cases. In Proceedings of the 5th International Congress of Mathematicians, Cambridge, UK, 22-28 August 1912; pp. $195-208$.

3. Beenakker, C.; Mazur, P. On the Smoluchowski Paradox in a Sedimenting Suspension. Phys. Fluids 1985, 28, 767-769. [CrossRef]

4. Batchelor, G.K. Sedimentation in a dilute dispersion of spheres. J. Fluid Mech. 1972, 52, 245. [CrossRef]

5. Beenakker, C.; Mazur, P. Is sedimentation container-shape dependent? Phys. Fluids 1985, 28, 3203. [CrossRef]

6. Caflisch, R.; Luke, J.H.C. Variance in the sedimentation speed of a suspension. Phys. Fluids 1985, 28, 759. [CrossRef]

7. Koch, D.L.; Shaqfeh, E. Screening in sedimenting suspensions. J. Fluid Mech. 1991, 224, 275. [CrossRef]

8. Ladd, A. Hydrodynamic screening in sedimenting suspensions of non-Brownian spheres. Phys. Rev. Lett. 1996, 76, 1392-1395. [CrossRef]

9. Ladd, A. Sedimentation of homogeneous suspensions of non-Brownian spheres. Phys. Fluids 1997, 9, 491-499. [CrossRef]

10. Cunha, F.R.; Sousa, A.J.; Hinch, E.J. Numerical Simulation of Velocity Fluctuations and Dispersion of Sedimentating Particles. Chem. Eng. Commun. 2002, 189, 1105-1129. [CrossRef]

11. Ladd, A.J.C. Effects of Container Walls on the Velocity Fluctuations of Sedimenting Spheres. Phys. Rev. Lett. 2002, 88, 048301. [CrossRef] [PubMed]

12. Brenner, M. Screening mechanisms in sedimentation. Phys. Fluids 1999, 11, 754. [CrossRef]

13. Mucha, P.J.; Tee, S.Y.; Weitz, D.A.; Shraiman, B.I.; Brenner, M. A model for velocity fluctuations in sedimentation. J. Fluid Mech. 2004, 501, 71-104. [CrossRef]

14. Nguyen, N.Q.; Ladd, A. Microstructure in a settling suspension of hard spheres. Phys. Rev. E 2004, 69, 050401. [CrossRef]

15. Nguyen, N.Q.; Ladd, A. Sedimentation of hard-sphere suspensions at low Reynolds number. J. Fluid Mech. 2005, 525, 73-104. [CrossRef]

16. Wang, J.; Schwarz, J.M.; Paulsen, J.D. Hyperuniformity with no fine tuning in sheared sedimenting suspensions. Nat. Commun. 2018, 9, 1-7. [CrossRef]

17. Yin, X.; Koch, D.L. Velocity fluctuations and hydrodynamic diffusion in finite-Reynolds-number sedimenting suspensions. Phys. Fluids 2008, 20, 043305. [CrossRef] 
18. Ramaswamy, S. Issues in the statistical mechanics of steady sedimentation. Adv. Phys. 2001, 50, $297-341$. 110050617. [CrossRef]

19. Guazzelli, E.; Hinch, J. Fluctuations and instability in sedimentation. Annu. Rev. Fluid Mech. 2010, 43, 97-116. [CrossRef]

20. Piazza, R. Settled and unsettled issues in particle settling. Rep. Prog. Physics. Phys. Soc. (Great Britain) 2014, 77, 056602. [CrossRef]

21. Cichocki, B.; Sadlej, K. Stokesian Dynamics-The BBGKY Hierarchy for Correlation Functions. J. Stat. Phys. 2008, $132,129-151$. [CrossRef]

22. Segrè, P.; Herbolzheimer, E.; Chaikin, P. Long-Range Correlations in Sedimentation. Phys. Rev. Lett. 1997, 79, 2574-2577. [CrossRef]

23. Segre, P.; Liu, F.; Umbanhowar, P.; Weltz, D. An effective gravitational temperature for sedimentation. Nature 2001, $409,594-597$. [CrossRef]

24. Bergougnoux, L.; Guazzelli, É. Dilute sedimenting suspensions of spheres at small inertia. J. Fluid Mech. 2021, 914, 1-19. [CrossRef]

25. Guazzelli, É. Evolution of particle-velocity correlations in sedimentation. Phys. Fluids 2001, 13, 1537-1540. [CrossRef]

26. Guazzelli, É. Sedimentation of small particles: How can such a simple problem be so difficult? Comptes Rendus-Mec. 2006, 334, 539-544. [CrossRef]

27. Miguel, M.C.; Pastor-Satorras, R. Velocity fluctuations and hydrodynamic diffusion in sedimentation. Europhys. Lett. 2001, 54, 45-50. [CrossRef]

28. Tee, S.Y.; Mucha, P.J.; Cipelletti, L.; Manley, S.; Brenner, M.P.; Segre, P.N.; Weitz, D.A. Nonuniversal velocity fluctuations of sedimenting particles. Phys. Rev. Lett. 2002, 89, 054501. [CrossRef] [PubMed]

29. Climent, E.; Maxey, M.R. Numerical simulations of random suspensions at finite Reynolds numbers. Int. J. Multiph. Flow 2003, 29, 579-601. [CrossRef]

30. Bergougnoux, L.; Ghicini, S.; Guazzelli, É.; Hinch, J. Spreading fronts and fluctuations in sedimentation. Phys. Fluids 2003, 15, 1875-1887. [CrossRef]

31. Gómez, D.C.; Bergougnoux, L.; Guazzelli, É.; Hinch, J. Fluctuations and stratification in sedimentation of dilute suspensions spheres. Phys. Fluids 2009, 21, 1-10. [CrossRef]

32. Gómez, D.C.; Bergougnoux, L.; Hinch, J.; Guazzelli, É. On stratification control of the velocity fluctuations in sedimentation. Phys. Fluids 2007, 19, 1-5. [CrossRef]

33. Kim, S.; Karilla, S.J. Microhydrodynamics: Principles and Selected Applications, Dover ed.; Dover Publications, Inc.: Mineola, NY, USA, 2005

34. Lamb, S.H. Hydrodynamics, 6th ed.; Cambridge University Press: Cambridge, UK, 1932.

35. Jones, R.B.; Schmitz, R. Mobility matrix for arbitrary spherical particles in solution. Phys. A Stat. Mech. Its Appl. 1988, 149, 373-394. [CrossRef]

36. Felderhof, B.U. Hydrodynamic interaction between two spheres. Phys. A Stat. Mech. Its Appl. 1977, 89, 373-384. [CrossRef]

37. Felderhof, B.U. Many-body hydrodynamic interactions in suspensions. Phys. A Stat. Mech. Its Appl. 1988, 151, 1-16. [CrossRef]

38. Cichocki, B.; Felderhof, B.U.; Hinsen, K.; Wajnryb, E.; Bławzdziewicz, J. Friction and mobility of many spheres in Stokes flow. J. Chem. Phys. 1994, 100, 3780. [CrossRef]

39. Szymczak, P.; Cichocki, B. A diagrammatic approach to response problems in composite systems. J. Stat. Mech. 2008, 2008, P01025. [CrossRef]

40. Schmitz, R.; Felderhof, B.U. Friction matrix for two spherical particles with hydrodynamic interaction. Phys. A Stat. Mech. Its Appl. 1982, 113, 103-116. [CrossRef]

41. Cichocki, B.; Felderhof, B.U.; Schmitz, R. Hydrodynamic interactions between two spherical particles. PCH PhysicoChemical Hydrodyn. 1988, 10, 383-403.

42. Felderhof, B.U.; Jones, R.B. Hydrodynamic scattering theory of flow about a sphere. Phys. A Stat. Mech. Its Appl. 1986, 136, 77-98. [CrossRef]

43. Felderhof, B.U.; Jones, R.B. Addition theorems for spherical wave solutions of the vector Helmholtz equation. J. Math. Phys. 1987, 28, 836-839. [CrossRef]

44. Felderhof, B.U.; Jones, R.B. Displacement theorems for spherical solutions of the linear Navier-Stokes equations. J. Math. Phys. 1989, 30, 339-342. doi.org/10.1063/1.528450. [CrossRef]

45. Ekiel-Jeżewska, M.; Wajnryb, E. Precise multipole method for calculating hydrodynamic interactions between spherical particles in the Stokes flow. In Theoretical Methods for Micro Scale Viscous Flows; Feuillebois, F., Sellier, A., Eds.; Transworld Research Network: Kerala, India, 2009; pp. 127-172.

46. Damour, T.; Iyer, B.R. Multipole analysis for electromagnetism and linearized gravity with irreducible Cartesian tensors. Phys. Rev. D 1991, 43, 3259-3272. [CrossRef] [PubMed]

47. Thorne, K.S. Multipole expansions of gravitational radiation. Rev. Mod. Phys. 1980, 52, 299-339. [CrossRef]

48. Balescu, R. Equilibrium and Nonequilibrium Statistical Mechanics; A Wiley-Interscience Publication: Hoboken, NJ, USA, 1975.

49. Batchelor, G.K. Sedimentation in a dilute polydisperse system of interacting spheres. Part 1. General Theory. J. Fluid Mech. 1982, 119, 379, doi.org/10.1017/S0022112082001402. [CrossRef]

50. Batchelor, G.K.; Wen, C.S. Sedimentation in a dilute polydisperse system of interacting spheres. Part 2. Numerical results. J. Fluid Mech. 1982, 124, 379. [CrossRef] 
51. Cichocki, B.; Sadlej, K. Steady-state particle distribution of a dilute sedimenting suspension. Europhys. Lett. 2005, 72, 936-942. [CrossRef]

52. Martin, P. Sum rules in charged fluids. Rev. Mod. Phys. 1988, 60, 1075-1127. [CrossRef]

53. Abade, G.C.; Cichocki, B. Sedimentation of non-Brownian suspensions. J. Phys. Conf. Ser. 2012, 392, 012002. [CrossRef] 